\section{High-speed label-free functional photoacoustic microscopy of mouse brain in action}

\author{
Junjie Yao ${ }^{1}$, Lidai Wang ${ }^{1}$, Joon-Mo Yang ${ }^{1}$, \\ Konstantin I Maslov ${ }^{1}$, Terence T W Wong ${ }^{1}$, Lei Li ${ }^{1}$, \\ Chih-Hsien Huang ${ }^{2}$, Jun Zou ${ }^{2} \&$ Lihong V Wang ${ }^{1}$
}

\begin{abstract}
We present fast functional photoacoustic microscopy (PAM) for three-dimensional high-resolution, high-speed imaging of the mouse brain, complementary to other imaging modalities. We implemented a single-wavelength pulse-width-based method with a one-dimensional imaging rate of $100 \mathrm{kHz}$ to image blood oxygenation with capillary-level resolution. We applied PAM to image the vascular morphology, blood oxygenation, blood flow and oxygen metabolism in both resting and stimulated states in the mouse brain.
\end{abstract}

Many biomedical imaging techniques, especially small-animal functional magnetic resonance imaging (fMRI), two-photon microscopy (TPM) and wide-field optical microscopy, have had profound impact on hemodynamic studies of the mouse brain by providing structural, blood oxygenation and flow dynamic information at various length scales. However, small-animal fMRI is insufficient to resolve brain hemodynamic activities at microscopic length scales finer than $50 \mu \mathrm{m}$ (ref. 1); phosphorescence lifetime-based TPM suffers from slow measurement of blood oxygenation ${ }^{2}$; and wide-field optical microscopy lacks depth resolution ${ }^{3}$.

Given these limitations, photoacoustic tomography (PAT) can play a complementary role. Previously reported PAT techniques variously lacked capillary-level resolution, wide-field imaging speed or blood oxygenation imaging capability ${ }^{4-8}$. Here, we present fast functional PAM, which is capable of high-resolution, highspeed imaging of the mouse brain through an intact skull in vivo. This PAM technology achieves a lateral spatial resolution of $\sim 3 \mu \mathrm{m}$ for structural imaging, which is five times finer than with our previous fast-scanning system ${ }^{7}, 25$ times finer than with our acousticresolution system ${ }^{6}$, and more than 35 times finer than with ultrasound array-based photoacoustic computed tomography ${ }^{5}$. Using a single-wavelength pulse-width-based method, PAM allows three-dimensional (3D) blood oxygenation imaging with capillary-level resolution at a 1D imaging rate of $100 \mathrm{kHz}$. PAM's blood oxygenation imaging speed is 50 times higher than with our fast-scanning PAM (ref. 8), 100 times higher than with our acoustic-resolution system ${ }^{6}$, and more than 500 times higher than with phosphorescence lifetime-based TPM ${ }^{2}$.

In PAM, both the excitation laser beams and the detection acoustic axis are confocally steered by a customized water-immersible MEMS (microelectromechanical system) scanning mirror (Fig. 1a, Supplementary Fig. 1 and Supplementary Note 1). The lateral resolution-perpendicular to the acoustic axis-is $\sim 3 \mu \mathrm{m}$ at the optical focus, and the axial resolution-along the acoustic axis-is $\sim 15 \mu \mathrm{m}$. The high laser pulse repetition rate of $500 \mathrm{kHz}$ enables dense sampling for morphological capillary-resolution imaging over a large scanning range. Our PAM method achieves a 2D frame rate of $400 \mathrm{~Hz}$ over an $\sim 3$-mm scanning range and a $3 \mathrm{D}$ volumetric rate of $1 \mathrm{~Hz}$ over a $3 \times 2 \mathrm{~mm}^{2}$ field of view (Fig. 1b).

By using a single-wavelength pulse-width-based method $\left(\mathrm{PW}-\mathrm{sO}_{2}\right)$, our PAM system can perform high-speed imaging of the oxygen saturation of hemoglobin $\left(\mathrm{sO}_{2}\right)$ (Fig. 1c). The two forms of hemoglobin, oxy- and deoxyhemoglobin $\left(\mathrm{HbO}_{2}\right.$ and $\mathrm{HbR}$, respectively), have different saturation intensities, defined as the excitation intensity that reduces the absorption coefficient to half its initial value ${ }^{9}$ (Supplementary Note 2 and Supplementary Fig. 2). When first excited by a picosecond pulse and subsequently by a nanosecond pulse of the same wavelength and pulse energy, $\mathrm{HbO}_{2}$ and $\mathrm{HbR}$ display different saturation levels (Supplementary Fig. 3). From photoacoustic (PA) signals acquired with the two laser pulses, the relative concentrations of $\mathrm{HbO}_{2}$ and $\mathrm{HbR}$ are quantified, and thus $\mathrm{sO}_{2}$ can be computed. $\mathrm{PW}-\mathrm{sO}_{2}$ does not suffer from wavelength-dependent optical attenuation as the traditional wavelength-tuning method does. Nevertheless, the maximum $\mathrm{PW}-\mathrm{sO}_{2}$ imaging depth is limited by optical attenuation to the point where saturation becomes insufficient.

To illustrate the structural imaging capabilities of our PAM system, we imaged a $5 \times 10 \mathrm{~mm}^{2}$ region of the mouse brain through an intact skull with the scalp removed (Fig. 1d and Supplementary Video 1) (acquisition time: $15 \mathrm{~s}$ ). We fixed the optical focal plane at $\sim 250 \mu \mathrm{m}$ beneath the skull surface. The imaging parameters for all the key experiments are summarized in Supplementary Table 1. By additional depth-scanning of the optical focal zone with a $z$-step size of $100 \mu \mathrm{m}$, PAM provided an imaging depth of $\sim 0.7 \mathrm{~mm}$ (Fig. 1e, Supplementary Fig. 4 and Supplementary Video 2), giving an effective pixel count of $\sim 47$ in focus along the depth direction. The optical scattering of brain tissue degraded the lateral resolution and image contrast of PAM with increasing imaging depth, as in any depth-resolved optical microscopy; thus, deep capillaries cannot be resolved by the current

${ }^{1}$ Optical Imaging Laboratory, Department of Biomedical Engineering, Washington University in St. Louis, St. Louis, Missouri, USA. ${ }^{2}$ Department of Electrical and Computer Engineering, Texas A\&M University, College Station, Texas, USA. Correspondence should be addressed to L.V.W. (lhwang@wustl.edu)

RECEIVED 25 APRIL 2014; ACCEPTED 20 FEBRUARY 2015; PUBLISHED ONLINE 30 MARCH 2015; D0I:10.1038/NMETH.3336 

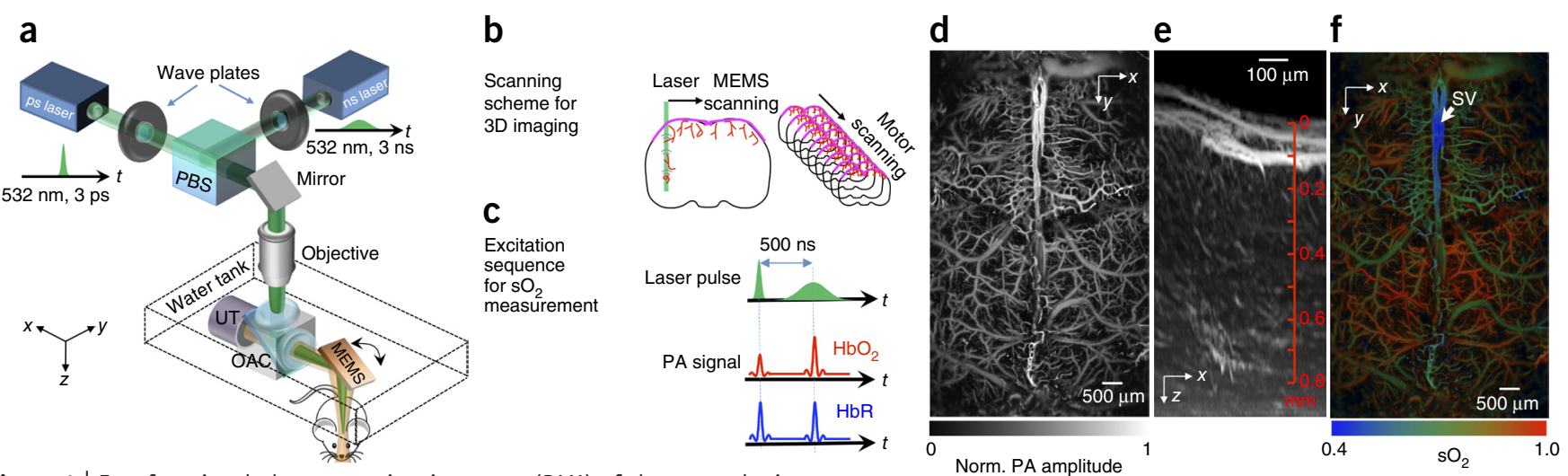

Figure 1 | Fast functional photoacoustic microscopy (PAM) of the mouse brain.

(a) Schematic of the PAM system. OAC, optical-acoustic combiner; PBS, polarizing beam splitter; UT, ultrasonic transducer. (b) Scheme of PAM scanning. 3D imaging is achieved by fast MEMS mirror scanning along the $x$ axis and slow motor-stage scanning along the $y$ axis. (c) Sequence of PAM excitation and detection. The picosecond pulse incident on oxyhemoglobin $\left(\mathrm{HbO}_{2}\right)$ results in more saturation and thus a weaker photoacoustic amplitude (PA) signal than the following nanosecond pulse, whereas the difference for deoxyhemoglobin (HbR) is negligible.

(d) Representative $x y$ projected brain vasculature image through an intact skull $(n=6)$.

(e) Representative enhanced $x z$ projected brain vasculature image acquired over a $0.6 \times 0.6 \mathrm{~mm}^{2}$

region with depth scanning, where the signal amplitude was normalized depthwise $(n=6)$. (f) PAM of

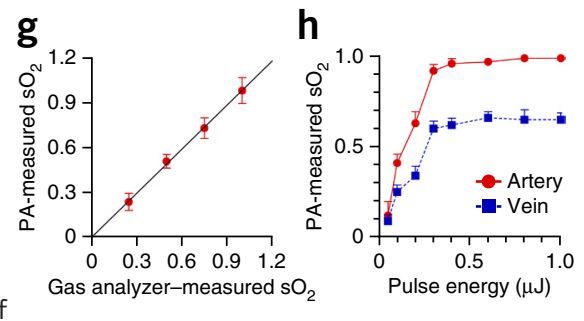
oxygen saturation of hemoglobin $\left(\mathrm{s}_{2}\right)$ in the same mouse brain as in $\mathbf{d}$, acquired by using the single-wavelength pulse-width-based method ( $\mathrm{PW}^{-\mathrm{s} \mathrm{O}_{2}}$ ) with two lasers. SV, skull vessel. (g) Comparison of the $\mathrm{PW}-\mathrm{sO}_{2}$ measurements in four blood phantoms with gas analyzer readings. The data are averaged over ten measurements on each phantom; error bars, s.d. (h) In vivo PW- $\mathrm{s}_{2}$ measurements in an artery-vein pair in a mouse ear with varied excitation pulse energies. The data are averaged over ten measurements on a mouse; error bars, s.d.

version of PAM. We confirmed PAM of the brain vasculature by TPM (Supplementary Fig. 5). A few vessels were revealed by TPM but not by PAM, probably owing to the lack of red blood cell (RBC) perfusion ${ }^{10}$. The acoustic sectioning of PAM could not resolve the blood vessels along the $z$ axis as well as the optical sectioning of TPM. The skull degraded the image quality of PAM by blurring the optical focusing and attenuating the PA signal (Supplementary Note 3 and Supplementary Figs. 6 and 7).

We mapped $\mathrm{sO}_{2}$ of the mouse brain vessel by vessel using the pulse-width-based method (Fig. 1f) (acquisition time: $\sim 40 \mathrm{~s}$ ). Unless otherwise stated, the pulse energy for all the $\mathrm{sO}_{2}$ measurements was $400 \mathrm{~nJ}$, and the estimated optical fluence at the optical focus was $\sim 0.3 \mathrm{~J} / \mathrm{cm}^{2}$. We used the nonsaturated PA signal acquired with nanosecond excitation to correct for optical attenuation and the laser spot size. The averaged $\mathrm{sO}_{2}$ level observed in the skull vessels was lower than that in the cortical vessels, a result consistent with the low-oxygenation microenvironment in bone marrow ${ }^{11}$.

We validated the $\mathrm{PW}-\mathrm{sO}_{2}$ method on blood phantoms, with an average measurement error (s.e.m.) of $2.7 \%$ (Fig. 1g), and we also compared the method with the traditional two wavelength-based method in vivo, finding an average difference of $<5 \%$ for superficial vessels $^{4}$ (Supplementary Fig. 8). To quantify the underestimation of $\mathrm{sO}_{2}$ by $\mathrm{PW}-\mathrm{sO}_{2}$ induced by light attenuation with increasing depth, we measured the $\mathrm{sO}_{2}$ in blood phantoms and in a mouse ear in vivo with pulse energies from $50 \mathrm{~nJ}$ to $1,000 \mathrm{~nJ}$ (Fig. $\mathbf{1 h}$ and Supplementary Figs. 9 and 10). When the pulse energy was $300 \mathrm{~nJ}$, the measurement error was $\sim 3 \%$ for absolute PW-sO $\mathrm{S}_{2}$ measurements.

We carefully investigated the potential for tissue damage induced by PAM. First, bright-field microscopy of a single layer of mouse RBCs, before and after PAM imaging ex vivo, confirmed that the PAM-imaged RBCs were intact, showing a donut-shaped morphology (Supplementary Fig. 11). Second, TPM of a mouse brain after PAM imaging with the laser pulse energy intentionally increased to 1,000 nJ ruled out PAM's potential to induce bleeding (Supplementary Fig. 12a). Last, standard hematoxylin and eosin (H\&E) histology on a mouse brain after PAM imaging showed no burn damage to the brain tissue (Supplementary Fig. 12b). As a positive control, we intentionally burned a part of the brain and studied it histologically. We compared representative histological slices from the inside and outside of the burned area, as well as the imaged area, and observed no burns in the imaged area (Supplementary Fig. 13).

Directly imaging hyperemia in the brain can help advance understanding of neurovascular coupling. Here, we demonstrate the high-speed functional imaging capability of PAM by studying mouse cortical hemodynamic responses to electrical stimulations of the hindlimbs (Supplementary Fig. 1a). Upon stimulation, the PA amplitude in the contralateral somatosensory region increased until the end of the stimulation (Fig. 2a and Supplementary Video 3). Meanwhile, the ipsilateral somatosensory region followed a similar trend but responded more weakly (Supplementary Fig. 14a,b), a result suggesting vascular interconnection between the two hemispheres ${ }^{12}$. We also observed that the sagittal sinus region responded to both left and right hindlimb stimulations, possibly because it drains blood from both hemispheres simultaneously ${ }^{12}$. The depth-resolved responses revealed that the responding region covered a depth range of $50-150 \mu \mathrm{m}$ beneath the cortical surface (Fig. 2b). The amplitude responses from the deep capillary beds were stronger than those from the major arteries and veins ${ }^{3}$ (Supplementary Fig. 15a,b).

Meanwhile, the artery dilated substantially in the contralateral hemisphere during the stimulations (Supplementary Figs. 14c and 15a). In the ipsilateral somatosensory region, arterial dilation was also observed but with a much weaker magnitude 
Figure 2 | PAM of brain responses to electrical stimulations of the hindlimbs of mice $(n=6)$. (a) Fractional photoacoustic amplitude changes (shown in yellow) in response to left hindlimb stimulation (LHS) and right hindlimb stimulation (RHS), superimposed on the vascular image (shown in red).

$\mathrm{LH}$, left hemisphere; $\mathrm{RH}$, right hemisphere.

(b) Depth-resolved photoacoustic amplitude (PA) responses. The responding areas in the $\mathrm{LH}$ and $\mathrm{RH}$ are shown in red and blue, respectively, and superimposed on the grayscale $y z$ projection image. The signal amplitude in the $y z$ projection image was normalized depthwise. (c) Fast $\mathrm{SO}_{2}$ imaging before (left) and during (right) stimulations of the left hindlimb. Three $0.3 \times$ $0.3 \mathrm{~mm}^{2}$ subregions ( $i$, ii and iii) are further analyzed (Supplementary Fig. 17).

(d) Time courses of the fractional changes in the cerebral blood flow (CBF), oxygen extraction

fraction $(\mathrm{OEF})$ and cerebral metabolic rate of oxygen $\left(\mathrm{CMRO}_{2}\right)$ in the core responding region. The gray bars outline the stimulation periods. All the $\mathrm{s}_{2}$ measurements were acquired with two lasers. The data in $\mathbf{d}$ are averaged over five trials on each of six mice; error bars, s.e. The measured $\mathrm{CBF}$, $0 \mathrm{EF}$ and $\mathrm{CMRO}_{2}$ are statistically compared for resting versus stimulated states. ${ }^{* *} P<0.001$, calculated by paired Student's $t$-test.

(Supplementary Fig. 14d). Veins did not show dilations ${ }^{3}$ (Supplementary Fig. 15a,c). Deep capillary beds are reported to dilate less than $0.5 \mu \mathrm{m}$ in diameter ${ }^{13}$, which is not resolvable by the current version of PAM. We repeated fast line scanning along the vessel axis to measure the blood flow speed ${ }^{8,14}$ (Supplementary Fig. 16). Stimulations induced a substantial increase in blood flow speed in both arteries and veins ${ }^{14}$ (Supplementary Figs. 14e and 15d). However, PAM could not detect the flow speed changes in deep capillaries.

Upon stimulation, $\mathrm{sO}_{2}$ levels increased substantially in veins and deep capillary beds (Fig. $2 \mathrm{c}$ and Supplementary Videos $\mathbf{4}$ and 5). The fractional change in $\mathrm{sO}_{2}$ diminished with increasing distance from the core responding region (Supplementary Fig. 17a,b), which was $\sim 100 \mu \mathrm{m}$ below the cortical surface ${ }^{3}$ (Supplementary Fig. 17c). The $\mathrm{sO}_{2}$ increase was greater in deep capillary beds than in veins and was insignificant in arteries (Supplementary Fig. 15e). The lack of arterial $\mathrm{sO}_{2}$ response is consistent with the fact that arterial blood has not yet reached capillaries for oxygen consumption and thus maintains a high oxygenation level ${ }^{3}$.

In the core responding region, the increase in $\mathrm{sO}_{2}$ in veins also manifested as a decrease in the oxygen extraction fraction ${ }^{15}$ (Fig. 2d). The fractional change in the cerebral metabolic rate of oxygen $\left(\mathrm{CMRO}_{2}\right)$ can be estimated from the above hemodynamic measurements (Online Methods). We observed a moderate fractional increase in $\mathrm{CMRO}_{2}$, peaking at $\sim 15 \%$ (Fig. 2d). The ratio between the fractional changes in cerebral blood flow $(\mathrm{CBF})$ and $\mathrm{CMRO}_{2}$ (i.e., the flow-consumption ratio) was $\sim 2.0$, which is consistent with the literature ${ }^{16}$.

In summary, using endogenous contrast, PAM can perform high-speed, high-resolution imaging of the vascular morphology, blood oxygenation, blood flow dynamics and oxygen metabolism of the mouse brain. In particular, PAM has achieved a 1D time-resolved imaging rate of $500 \mathrm{kHz}$ for morphological imaging and $100 \mathrm{kHz}$ for blood oxygenation imaging. In the future, the axial resolution of PAM can be improved by using an ultrasonic transducer with a wider bandwidth or by using nonlinear PA mechanisms ${ }^{17}$. Near-infrared wavelengths can increase the imaging depth of $\mathrm{PAM}^{17}$. The potential for optical breakdown in
RBCs in vivo, which is relevant to excitation pulse width, can be further investigated by using photothermal microscopy or other methods ${ }^{18}$.

Briefly, the advantages and limitations of PAM for mouse brain hemodynamic imaging are as follows. (i) PAM provides better depth resolution and greater absorption-based image contrast than wide-field optical microscopy but is slower and more expensive. (ii) In contrast with TPM, PAM does not need exogenous contrast agents and point-by-point depth scanning, but PAM has worse axial resolution and, at the currently available wavelengths, less penetration. (iii) Whereas fMRI is sensitive only to HbR, PAM is sensitive to both $\mathrm{HbR}$ and $\mathrm{HbO}_{2}$, and PAM has higher spatial and temporal resolutions; however, fMRI can provide deeper penetration. In sum, PAM is highly complementary to other brain imaging modalities in its contrast mechanism, spatiotemporal resolution and functional imaging capability.

\section{METHODS}

Methods and any associated references are available in the online version of the paper.

Note: Any Supplementary Information and Source Data files are available in the online version of the paper.

\section{ACKNOWLEDGMENTS}

The authors appreciate J. Ballard's close reading of the manuscript. We thank C. Li, C. Chen, W. Chapman and J. Lee for discussions and technical support. This work was sponsored by US National Institutes of Health (NIH) grants DP1 EB016986 (NIH Director's Pioneer Award), R01 CA186567 (NIH Director's Transformative Research Award), 1S10 RR028864 and R01 CA159959 (to L.V.W.), and US National Science Foundation (NSF) grant CMMI-1131758 (to J.Z.). The bright-field microscopy was performed at the Alafi Neuroimaging Laboratory of the Hope Center for Neurological Disorders, Washington University School of Medicine, which is supported by the NIH Neuroscience Blueprint Center Core grant P30 NS057105.

\section{AUTHOR CONTRIBUTIONS}

J.Y., J.Z. and L.V.W. conceived of and designed the study. J.Y., J.-M.Y., K.I.M. L.W. and C.-H.H. constructed the imaging system. J.Y., T.T.W.W. and L.L. performed the experiments and analyzed the data. J.Y., L.W. and L.V.W. wrote the manuscript. L.V.W. supervised the whole study. All authors discussed the conceptual and practical implications of the methods. 


\section{COMPETING FINANCIAL INTERESTS}

The authors declare competing financial interests: details are available in the online version of the paper.

Reprints and permissions information is available online at http://www.nature com/reprints/index.html.

1. Kim, D.S., Duong, T.Q. \& Kim, S.G. Nat. Neurosci. 3, 164-169 (2000).

2. Sakadzić, S. et al. Nat. Methods 7, 755-759 (2010).

3. Hillman, E.M.C. et al. Neuroimage 35, 89-104 (2007).

4. Hu, S., Maslov, K., Tsytsarev, V. \& Wang, L.V. J. Biomed. Opt. 14, 040503 (2009).

5. Deán-Ben, X.L. \& Razansky, D. Light Sci. Appl. 3, e137 (2014).

6. Stein, E.W., Maslov, K. \& Wang, L.H.V. J. Biomed. Opt. 14, 020502 (2009).

7. Yao, J. et al. J. Biomed. Opt. 17, 080505 (2012).

8. Wang, L., Maslov, K. \& Wang, L.V. Proc. Natl. Acad. Sci. USA 110, 5759-5764 (2013).
9. Danielli, A., Favazza, C.P., Maslov, K. \& Wang, L.V. Opt. Lett. 36, 769-771 (2011).

10. Yao, J., Maslov, K., Hu, S. \& Wang, L.V. J. Biomed. Opt. 14, 054049 (2009).

11. Spencer, J.A. et al. Nature 508, 269-273 (2014)

12. Liao, L.D. et al. J. Cereb. Blood Flow Metab. 32, 938-951 (2012).

13. Villringer, A., Them, A., Lindauer, U., Einhäupl, K. \& Dirnagl, U. Circ. Res. 75, 55-62 (1994).

14. Shih, A.Y. et al. J. Cereb. Blood Flow Metab. 32, 1277-1309 (2012).

15. Ogawa, S., Lee, T.M., Kay, A.R. \& Tank, D.W. Proc. Natl. Acad. Sci. USA 87, 9868-9872 (1990).

16. Dunn, A.K., Devor, A., Dale, A.M. \& Boas, D.A. Neuroimage 27, 279-290 (2005).

17. Wang, L., Xia, J., Yao, J., Maslov, K.I. \& Wang, L.V. Phys. Rev. Lett. 111, 204301 (2013).

18. Lukianova-Hleb, E.Y., Oginsky, A.0., Olson, J.S. \& Lapotko, D.0. Lasers Surg. Med. 43, 249-260 (2011). 


\section{ONLINE METHODS}

Photoacoustic tomography (PAT). In PAT, as photons travel in tissue, some are absorbed by biomolecules, and their energy is partially or completely converted into heat. The heat-induced pressure wave propagates in tissue and is detected outside the tissue by an ultrasonic transducer or transducer array to form an image that maps the original optical energy deposition in the tissue. PAT has a $100 \%$ relative sensitivity to optical absorption, which means a given percentage change in the optical absorption coefficient yields the same percentage change in the PA signal amplitude. In PAT operating at $532 \mathrm{~nm}$, the high contrast of cortical microvasculature comes from the absorption of hemoglobin in red blood cells (RBCs), which overpowers the absorption of other absorbers such as water and lipids by three orders of magnitude. As the optical absorption of blood is highly oxygenation dependent, PAT can measure blood oxygenation with high sensitivity. Photoacoustic microscopy (PAM) is a microscopic focusedscanning embodiment of PAT.

Fast functional photoacoustic microscopy. To induce photoacoustic signals, we combine a $3-\mathrm{ns}$ pulsed laser beam at $532 \mathrm{~nm}$ (AOT-YVO-100Q, AOT Inc.; pulse repetition rate: $100 \mathrm{kHz}$ ) with a 3-ps pulsed laser beam at $532 \mathrm{~nm}$ (APL-4000-1064, RPMC Lasers, Inc.; pulse repetition rate: $500 \mathrm{kHz}$ ) via a polarizing beam splitter (PBS251, Thorlabs, Inc.). The polarizations of the two laser beams are adjusted by wave plates to maximize the combining efficiency. Laser energy fluctuations are monitored by a fast photodiode that samples a small portion of the laser beams. The laser beams are focused by a plano-convex lens (LA1131, Thorlabs, Inc.) and then spatially filtered by a $50-\mu \mathrm{m}$-diameter pinhole (P50C, Thorlabs, Inc.). The filtered laser beams are focused to $\sim 3-\mu \mathrm{m}$ spots by an objective lens (AC127-050-A, Thorlabs, Inc.; NA: 0.1 in air). The optical focal zone (also known as the depth of focus), defined as the full-width at half-maximum of the peak intensity, is $\sim 83 \mu \mathrm{m}$, within which the lateral resolution degrades up to $\sqrt{2}$; correspondingly, the depth range within which the lateral resolution degrades up to a factor of 2 is $\sim 144 \mu \mathrm{m}$. An optical-acoustic beam combiner, composed of an aluminum-coated prism (NT32331, Edmund, Inc.) and an uncoated prism (NT32-330, Edmund, Inc.), provides optical-acoustic coaxial alignment. Here, the thin aluminum coating reflects light but transmits sound. An optical correction lens attached to the top surface of the combiner corrects the optical aberration due to the prism. The focused laser beams and the generated photoacoustic waves are both directed by a MEMS scanning mirror in a coaxial configuration. The photoacoustic waves are focused by an acoustic lens and detected by an ultrasonic transducer (V214-BB-RM, Olympus-NDT, Inc.; central frequency: $50 \mathrm{MHz}$; one-way $-6 \mathrm{~dB}$ bandwidth: 100\%).

Driven by sufficiently strong electromagnetic force, the whole MEMS scanning mirror can operate under deionized water in a water tank, which is required to provide acoustic coupling from the sample surface to the acoustic lens. The bottom of the water tank is sealed with a piece of membrane that is both optically and acoustically transparent. In PAM, because the acoustic flight time provides depth information along the acoustic axis, each excitation laser pulse generates a $1 \mathrm{D}$ depth-resolved image. Volumetric imaging is provided by fast angular scanning of the MEMS mirror along the $x$ axis and slow linear motor-stage scanning of the sample along the $y$ axis at a speed of $2-4 \mathrm{~mm} / \mathrm{s}$. In PAM, a 3- $\mu \mathrm{m}$ lateral resolution at the optical focus and a $15-\mu \mathrm{m}$ axial resolution have been achieved in clear media. The axial resolution of PAM is jointly determined by the laser pulse width, the frequencydependent acoustic attenuation in tissue, and the frequency response of the ultrasonic transducer. The maximum in-focus scanning range is $\sim 3.0 \mathrm{~mm}$ along the $x$ axis, with a cross-sectional frame rate of $400 \mathrm{~Hz}$. When necessary, additional depth-scanning of the optical focal zone extends the focal range of PAM at the expense of imaging speed. By steering both the optical and acoustic axes simultaneously, PAM maintains confocal alignment and high detection sensitivity over the field of view. The two lasers are triggered with a time interval of $500 \mathrm{~ns}$. The 500-ns delay allows the first PA signal to travel $0.75 \mathrm{~mm}$, which is approximately the maximum penetration depth of PAM in the brain.

PAM of oxygen saturation $\left(\mathrm{sO}_{2}\right)$. In PAM, the two lasers emit the same pulse energy at $532 \mathrm{~nm}$. Because the picosecond pulse has a higher peak intensity than the nanosecond pulse, it results in more saturation (Supplementary Fig. 3a,b). We define a saturation factor as the ratio of the photoacoustic amplitudes under picosecond and nanosecond excitations (Supplementary Fig. 3c).

The relative concentrations of $\mathrm{HbR}$ and $\mathrm{HbO}_{2}$ can be estimated by solving the following linear equations:

$$
\begin{gathered}
P_{\mathrm{ns}}=\ln (10) k F\left(\varepsilon_{\mathrm{HbR}} C_{\mathrm{HbR}}+\varepsilon_{\mathrm{HbO}_{2}} C_{\mathrm{HbO}_{2}}\right) \\
P_{\mathrm{ps}}=\ln (10) k F\left(r_{\mathrm{HbR}}(F) \varepsilon_{\mathrm{HbR}} C_{\mathrm{HbR}}+r_{\mathrm{HbO}_{2}}(F) \varepsilon_{\mathrm{HbO}_{2}} C_{\mathrm{HbO}_{2}}\right)
\end{gathered}
$$

where $P_{\mathrm{ns}}$ and $P_{\mathrm{ps}}$ are the PA amplitudes under nanosecond and picosecond excitations, respectively; $k$ is a proportionality coefficient related to the detection system, the Grüneisen parameter, and the nonradiative quantum yield; $\varepsilon_{\mathrm{HbR}}$ and $\varepsilon_{\mathrm{HbO}_{2}}$ are the molar extinction coefficients of $\mathrm{HbR}$ and $\mathrm{HbO}_{2}$, respectively; $C_{\mathrm{HbR}}$ and $\mathrm{C}_{\mathrm{HbO}_{2}}$ are the molar concentrations of $\mathrm{HbR}$ and $\mathrm{HbO}_{2}$, respectively; and $r_{\mathrm{HbR}}$ and $r_{\mathrm{HbO}_{2}}$ are the saturation factors of $\mathrm{HbR}$ and $\mathrm{HbO}_{2}$, respectively, which are functions of the local fluence $F$ (i.e., the photon energy imposed over a unit area).

Note since $\varepsilon_{\mathrm{HbR}} \approx \varepsilon_{\mathrm{HbO}_{2}}$ at $532 \mathrm{~nm}$ and $r_{\mathrm{HbR}} \approx 1$, equations (1) and (2) are reduced to

$$
\begin{gathered}
P_{\mathrm{ns}} \approx k_{1}\left(C_{\mathrm{HbR}}+C_{\mathrm{HbO}_{2}}\right) \\
P_{\mathrm{ps}} \approx k_{1}\left(C_{\mathrm{HbR}}+r_{\mathrm{HbO}_{2}}(F) C_{\mathrm{HbO}_{2}}\right)
\end{gathered}
$$

where $k_{1}=\ln (10) k \varepsilon_{\mathrm{HbR}} F$. Once equations (3) and (4) are solved, the total hemoglobin concentration $C_{\mathrm{HbT}}$ is computed by

$$
C_{\mathrm{HbT}}=C_{\mathrm{HbR}}+C_{\mathrm{HbO}_{2}}
$$

Therefore, $\mathrm{sO}_{2}$ can be computed as

$$
\mathrm{sO}_{2}=\frac{C_{\mathrm{HbO}_{2}}}{C_{\mathrm{HbT}}} \approx \frac{1-\frac{P_{\mathrm{ps}}}{P_{\mathrm{ns}}}}{1-r_{\mathrm{HbO}_{2}}(F)}=k_{2}\left(1-\frac{P_{\mathrm{ps}}}{P_{\mathrm{ns}}}\right)
$$

where

$$
k_{2}=\frac{1}{1-r_{\mathrm{HbO}_{2}}(F)}
$$


From equation (5), we can see that $r_{\mathrm{HbO}_{2}}$ is needed for absolute $\mathrm{sO}_{2}$ measurement. $r_{\mathrm{HbO}_{2}}$ is estimated using the local fluence $F$ (Supplementary Fig. 3c), which is proportional to the unsaturated photoacoustic signal $P_{\mathrm{ns}}$ (Supplementary Fig. $3 \mathbf{b}$ ). Note that the local fluence change due to the varied laser spot size at different depth is also accounted for by $P_{\mathrm{ns}} \cdot r_{\mathrm{HbO}_{2}}$ can also be estimated on the basis of the neighboring arteries.

In studies where only fractional changes in $\mathrm{sO}_{2}$ are of interest, we have

$$
\frac{\Delta \mathrm{sO}_{2}}{\mathrm{sO}_{2}} \approx \frac{\Delta\left(\frac{P_{\mathrm{ps}}}{P_{\mathrm{ns}}}\right)}{1-\frac{P_{\mathrm{ps}}}{P_{\mathrm{ns}}}}
$$

Equation (6) shows that local fluence is not needed for measuring fractional changes in $\mathrm{sO}_{2}$, as long as the saturation is sufficient.

PAM of cerebral metabolic rate of oxygen $\left(\mathrm{CMRO}_{2}\right)$. If the cortical region of interest has well-defined feeding arteries and draining veins, and the volumetric blood flow rates in the feeding and draining vessels are conserved, $\mathrm{CMRO}_{2}$ can be calculated as

$$
\mathrm{CMRO}_{2}=\xi \times\left(\mathrm{sO}_{2 \text {-artery }}-\mathrm{sO}_{2 \text {-vein }}\right) \times C_{\mathrm{HbT}} \times \mathrm{CBF} / W
$$

where $\xi$ is the oxygen binding capacity of hemoglobin $\left(1.36 \mathrm{~mL} \mathrm{O}_{2}\right.$ per gram hemoglobin or $87.7 \mathrm{l}_{2}$ per mol hemoglobin); $\mathrm{sO}_{2 \text {-artery }}$ and $\mathrm{sO}_{2 \text {-vein }}$ are the oxygen saturation averaged in the artery and vein, respectively; $\mathrm{CBF}$ is the volumetric blood flow rate $(\mathrm{L} / \mathrm{s})$; and $W$ is the weight of the region of interest (in grams).

Oxygen extraction fraction (OEF) is defined as the fractional difference between the arterial and venous oxygen saturation:

$$
\mathrm{OEF}=\frac{\mathrm{sO}_{2 \text {-artery }}-\mathrm{sO}_{2 \text {-vein }}}{\mathrm{sO}_{2 \text {-artery }}}
$$

Under normal conditions, arterial blood is close to fully oxygenated (i.e., $\mathrm{sO}_{2 \text {-artery }} \approx 1$ ). We can rewrite equation (7) as

$$
\begin{aligned}
\mathrm{CMRO}_{2} & =\xi \times \mathrm{OEF} \times \mathrm{sO}_{2 \text {-artery }} \times C_{\mathrm{HbT}} \times \mathrm{CBF} / W \\
& \approx \xi \times \mathrm{OEF} \times C_{\mathrm{HbT}} \times \mathrm{CBF} / W
\end{aligned}
$$

Because the volumetric blood flow rates are conserved, we have

$$
\mathrm{CBF}=\frac{\pi}{4}\left(d_{\text {vein }}\right)^{2} v_{\text {vein }}
$$

where $d_{\text {vein }}$ is the diameter of the vein, and $v_{\text {vein }}$ is the average blood flow speed in the vein.

Assuming $W$ does not change during the brain activity, we have

$$
\begin{aligned}
1+\frac{\Delta \mathrm{CMRO}_{2}}{\mathrm{CMRO}_{2}} & \approx\left(1+\frac{\Delta \mathrm{OEF}}{\mathrm{OEF}}\right)\left(1+\frac{\Delta \mathrm{CBF}}{\mathrm{CBF}}\right)\left(1+\frac{\Delta C_{\mathrm{HbT}}}{C_{\mathrm{HbT}}}\right) \\
& \approx\left(1-\frac{\Delta \mathrm{sO}_{2 \text {-vein }}}{\mathrm{sO}_{2 \text {-vein }}}\right)\left(1+\frac{\Delta d_{\text {vein }}}{d_{\text {vein }}}\right)^{2}\left(1+\frac{\Delta v_{\text {vein }}}{v_{\text {vein }}}\right)\left(1+\frac{\Delta C_{\mathrm{HbT}}}{C_{\mathrm{HbT}}}\right)
\end{aligned}
$$

From equation (11), we can calculate the fractional change in $\mathrm{CMRO}_{2}$ from the fractional changes in other parameters measured individually.
Experimental animals. Six female ND4 Swiss Webster mice (Harlan Laboratory, Inc.; 16-30 g, 3-10 weeks old) were used for the studies. The laboratory animal protocols were approved by the Animal Studies Committee of Washington University in St. Louis. During the experiment, the mouse's temperature was kept at $37^{\circ} \mathrm{C}$ by a water-circulating heating pad. An intraperitoneal dose of $100 \mathrm{mg}$ per kg body weight $\alpha$-chloralose was used for anesthesia, which had a relatively mild effect on brain functions ${ }^{14}$. The mouse was then taped to a lab-made animal holder, which was mounted to the PAM system. The head of the mouse was fixed in a stereotaxic frame. Before imaging, the scalp was surgically removed; the skull was left intact. Bloodstains on the skull surface-even when invisible to the naked eye-could generate strong photoacoustic signals; thus, the exposed skull surface was carefully cleaned with phosphate buffered saline (PBS) solution. Ultrasound gel was then applied on the skull surface to retain moisture and couple the acoustic signals. A water tank filled with deionized water was then placed on top of the mouse head. The membrane at the bottom of the water tank was in gentle contact with the ultrasound gel. The translation of the animal holder by the motor stage at a speed of $2-4 \mathrm{~mm} / \mathrm{s}$ did not induce significant disturbance to the animal and the water in the tank.

Electrical stimulations to hindlimbs. Electrical stimulations were introduced by two pairs of needle electrodes inserted under the skin of the right and left hindlimbs, respectively. The electrodes were connected to a function generator (DS345, Stanford Research Systems, Inc.) through a manual switch. The whole procedure consisted of five periods and lasted for $5 \mathrm{~min}$. The first, third, and fifth periods were resting states, whereas the second period, from $60 \mathrm{~s}$ to $80 \mathrm{~s}$, was left hindlimb stimulation, and the fourth period, from 200 s to 220 s, was right hindlimb stimulation. Each stimulation period consisted of a train of electrical pulses with an amplitude of $2 \mathrm{~mA}$, a pulse width of $0.25 \mathrm{~ms}$, and a repetition rate of $2 \mathrm{~Hz}$. The stimulation period and intensity were controlled without inducing any paw motions. Five trials were performed on each mouse.

Automatic vessel segmentation. The PA signal amplitude was extracted through the Hilbert transformation of each 1D depthresolved signal. The data were then processed using a customized vessel-segmentation algorithm. All the data analysis was performed in three dimensions. Briefly, a cross-sectional image was first converted to a binary image, where the cross-section of each vessel was identified and labeled. By tracking the cross-sections of each vessel throughout all the cross-sectional images, we could individually label and thus segment the vessels. All the segmented vessels were visually evaluated and corrected if necessary. The final segmentation information was then stored for future use. The vessel segmentation algorithm can be performed along different orientations. The computer code is available on request.

The vessel segmentation was used to measure blood vessel diameters and correct the $\mathrm{sO}_{2}$ calculation. To measure the vessel diameter, we measured the length of a line across the vessel at different angles relative to the vessel's axis and chose the shortest path length as the vessel diameter. To correct the $\mathrm{sO}_{2}$ calculation, we separated any overlapping vessels at the junction through segmentation, and the $\mathrm{sO}_{2}$ of each vessel was calculated individually. 
PAM of blood flow speed. Line scanning along the axis of a vessel can be used to measure the blood flow speed. Moving RBCs imaged at a sufficient rate appear as bright-dark streaks in the resulting space-time map. The slope of the bright-dark streaks, measured from the vertical direction, is proportional to the centerline (axial) flow speed. The slope is measured by using a 2D Fourier transformation of the space-time map. The flow direction can be determined from the sign of the slope and the direction of the line-scan sweep. To measure high flow speeds more accurately, we imaged the same cells with at least five line scans. Therefore, the maximum measurable flow speed corresponds to a travel distance of $3 \mathrm{~mm}$ within $12.5 \mathrm{~ms}$, which translates to a flow speed of $\sim 24 \mathrm{~cm} / \mathrm{s}$.

H\&E histology. The right hemisphere of a mouse was imaged by PAM with a picosecond pulse energy of $1 \mu \mathrm{J}$ and a pulse repetition rate of $500 \mathrm{kHz}$. Immediately after the imaging, the mouse was transcardially perfused with $0.9 \%$ saline followed by $4 \%$ paraformaldehyde in PBS. The brain was removed and post-fixed in $4 \%$ paraformaldehyde for $24 \mathrm{~h}$. Coronal sections ( $5 \mu \mathrm{m}$ thick) were cut, with paraffin embedding. Standard H\&E staining was performed on the sections, which were examined using bright-field microscopy (NanoZoomer, Hamamatsu) with a $20 \times$ objective $(\mathrm{NA}=0.67)$. In the positive control experiment, the left hemisphere of a mouse was illuminated by a continuous-wave laser (GM-CF02-100, Information Unlimited, Inc.; wavelength: $532 \mathrm{~nm}$; power: $100 \mathrm{~mW}$; spot size: $\sim 0.25 \mathrm{~mm}^{2}$ ) for 1 min to induce thermal coagulation (i.e., burn). The right hemisphere was imaged by PAM for $1 \mathrm{~min}$ with a pulse energy of $1 \mu \mathrm{J}$ and a pulse repetition rate of $500 \mathrm{kHz}$. $\mathrm{H} \& \mathrm{E}$ histology was then performed on the brain with the same procedure as above.

Two-photon microscopy imaging. To rule out the potential for causing vessel leakage by PAM, we imaged a mouse with twophoton microscopy (Fluoview 1000, Olympus, Inc.) after PAM imaging (pulse energy: $1 \mu \mathrm{J}$; pulse repetition rate: $500 \mathrm{kHz}$ ). For two-photon microscopy, the skull was thinned to $\sim 30 \mu \mathrm{m}$ using a dental drill and a microsurgical blade as previously described ${ }^{14}$. FITC-dextran solution in PBS $(150 \mu \mathrm{L}, 2.5 \% \mathrm{w} / \mathrm{v})$ was injected via a tail vein before two-photon imaging. A $4 \times$ objective $(\mathrm{NA}=0.10)$ was used to find the same imaging area as in PAM, and then a $20 \times$ objective (NA $=0.70)$ was used to acquire high-resolution images (excitation wavelength: $800 \mathrm{~nm}$; emission filter wavelength: 495-540 nm). Depth-scanning was performed from the skull surface to a depth of $600 \mu \mathrm{m}$ into the cortex, with a step size of $5 \mu \mathrm{m}$. The same procedure was also used for validating PAM with twophoton microscopy (Supplementary Fig. 5).

Reproducibility. The experiments were not randomized. The investigators were not blinded to allocation during experiments and outcome assessment. No sample-size estimation was performed to ensure adequate power to detect a prespecified effect size. 